\title{
GEN-FOAM MODEL AND BENCHMARK OF DELAYED NEUTRON PRECURSOR DRIFT IN THE MOLTEN SALT REACTOR EXPERIMENT
}

\author{
Jun Shi and Massimiliano Fratoni \\ University of California, Berkeley \\ Department of Nuclear Engineering, Berkeley, CA 94720, USA \\ junshi@berkeley.edu,maxfratoni@berkeley.edu
}

\begin{abstract}
The effective delayed neutron fraction is an important reactor kinetics parameter. In flowing liquid-fuel reactors, this differs from the delayed neutron fraction because of the emission of delayed neutrons with a lower energy spectrum than prompt and the delayed neutron precursor (DNP) drift due to the fuel movement. In general, neglecting delayed neutron precursor drift leads to an over-estimation of the effective delayed neutron fraction. Nevertheless, the capability to simulate this peculiar phenomenon is not available in most reactor physics tools. In this project, a multi-physics approach to modeling DNP drift is developed using the GeNFoam toolkit, and it benchmarked against available experimental data from the Molten Salt Reactor Experiment (MSRE). GeN-Foam couples a neutron diffusion solver with a thermalhydraulics solver. Additionally, a new function was added for solving adjoint multi-group diffusion eigenvalue problems and calculating effective delayed neutron fraction. For benchmarking, an R-Z model of the MSRE was developed in GeN-Foam. The porous media model was applied, and cross sections were generated using the Monte Carlo code Serpent-2 with ENDF/B-VII.1 nuclear data library. In order to evaluate the impact of DNP drift, two steady-state conditions (stationary and flowing salt at $1200 \mathrm{gpm}$ ) were simulated. A reactivity change of $-241 \mathrm{pcm}$ was calculated using GeN-Foam for the MSRE between static and flowing fuel, which is in a good agreement with the experimental value of $-212 \mathrm{pcm}$. The total effective delayed neutron fraction change was calculated to be $-230 \mathrm{pcm}$ vs. $-304 \mathrm{pcm}$ reported for the MSRE and analytical calculated during the experimental campaign. Three transient accidents were also analyzed.
\end{abstract}

KEYWORDS: GeN-Foam, Delayed Neutron Precursor Drift, Effective Delayed Neutron Fraction, Molten Salt Reactor Experiment (MSRE)

\section{INTRODUCTION}

Molten Salt Reactors (MSRs) are designed to be fail-safe and can provide a path towards clean and sustainable nuclear energy, and after decades of discontinuance, a renewed interest in this Generation IV reactor technology is observed. The modeling of delayed neutron precursor (DNP) circulation in liquid fuel MSRs is identified as one of the particular phenomena that are not available in traditional reactor physics tools. In order to address this need, we will use and further develop a toolset that couples $\mathrm{C}++$ multi-physics toolkit GeN-Foam [1] and the Monte Carlo code Serpent-2 [2]. 
The effective delayed neutron fraction ( $\left.\beta_{\text {eff }}\right)$ is an important reactor kinetics parameter. The contribution of delayed neutrons is of primary importance for the safe control of any nuclear reactor. In circulating-fuel reactors (e.g., molten salt reactors), the effective delayed neutron fraction differs from the delayed neutron fraction $\left(\beta_{0}\right)$ because of two distinct reasons. The first reason (common to solid-fuel reactors) is that the emission spectrum of delayed neutrons is softer than the of prompt neutrons: on average, the former are emitted with a lower energy. This may imply a difference in the importance of delayed and prompt neutrons. The second is that DNPs are transported by the fluid flow in the fuel circuit and may decay in positions of low importance and even out of the core. Spatial effects due to fuel motion are more relevant and always reduce the values of $\beta_{\text {eff. }}$ Energy effects are, in general, of less relevance and may reduce or increase the effective delayed neutron fraction, according to the neutronic characteristics of the core. Therefore, the movement of DNPs complicates the calculation of the effective delayed neutron fraction and the accurate modeling of reactor transient responses.

This paper summarizes the modeling of Molten Salt Reactor Experiment in a 2 dimensional-RZ (2D-RZ) geometry in GeN-Foam, with cross sections generated by Serpent-2 and ENDF/B-VII.1 nuclear data library. Two steady-state calculations (static and fuel flowing at $1200 \mathrm{gpm}$ ) are performed, and the obtained results are analyzed and compared with data from Oak Ridge National Laboratory (ORNL) MSRE reports [3-4]. Three transient behaviors (unprotected transient over power, unprotected loss of heat sink, and unprotected loss of flow) are also discussed, respectively.

\section{CALCULATION METHODOLOGY}

The GeN-Foam multi-physics solver is built upon four main components: a thermal-hydraulics sub-solver, a neutronics sub-solver, a thermal-mechanics sub-solver, and a sub-scale fuel sub-solver. In our current study, only the first two sub-solvers are used. Thus, the calculation methodologies of neutronics and thermal-hydraulics sub-solvers are very briefly presented here, and a more detailed explanation of GeNFoam code can be found in paper [1]. Note that all parameters are defined in section Nomenclature.

\subsection{Thermal-hydraulics Sub-solver}

The thermal-hydraulics sub-solver is based on the standard $k-\varepsilon$ turbulence model for compressible or incompressible flows but extended to coarse-mesh applications through the use of a porous medium approach for user-selected cell zones inside the mesh. In other words, fine mesh can be applied for simple structures (e.g., plenum region), and coarse mesh can be applied for complex structures (e.g., core region and heat exchanger) by treating them as porous media. The equations for the turbulent single-phase flow of a fluid in a porous medium can be derived from standard Navier-Stokes (NS) equations, which govern the motion of fluids and can be seen as Newton's second law of motion for fluids, via time and volume averages. The resulting equations of mass, momentum and energy conservation are [5-7]:

$$
\begin{gathered}
\quad \frac{\partial \gamma \rho}{\partial t}+\nabla \cdot\left(\rho \boldsymbol{u}_{\boldsymbol{D}}\right)=0, \\
\frac{\partial \rho \boldsymbol{u}_{\boldsymbol{D}}}{\partial t}+\frac{1}{\gamma} \nabla \cdot\left(\rho \boldsymbol{u}_{\boldsymbol{D}} \otimes \boldsymbol{u}_{\boldsymbol{D}}\right)=\nabla \cdot\left(\mu_{T} \nabla \boldsymbol{u}_{\boldsymbol{D}}\right)-\gamma \nabla p+\gamma \boldsymbol{F}_{\boldsymbol{g}}+\gamma \boldsymbol{F}_{s \boldsymbol{s}}-\left(\rho \boldsymbol{u}_{\boldsymbol{D}} \otimes \boldsymbol{u}_{\boldsymbol{D}}\right) \nabla \frac{1}{\gamma}, \\
\frac{\partial \gamma \rho e}{\partial t}+\nabla \cdot\left(\boldsymbol{u}_{\boldsymbol{D}}(\rho e+p)\right)=\gamma \nabla \cdot\left(k_{T} \nabla T\right)+\boldsymbol{F}_{\boldsymbol{s} \boldsymbol{s}} \cdot \boldsymbol{u}_{\boldsymbol{D}}+\gamma \dot{Q}_{s S}+\left(k_{T} \nabla T\right) \cdot \nabla \gamma,
\end{gathered}
$$

The appearance of a porosity $\gamma$ considers the fact that only part of the volume is occupied by the fluid, and two additional terms, $\mathbf{F}_{\text {ss }}$ and $\dot{\mathrm{Q}}_{\mathrm{ss}}$, represent the effects on fluid flow by the sub-scale structures. In case of clear fluid, by setting porosity to 1 and sub-scale structures' effects to 0 , these equations are converted back to the traditional Reynolds-averaged Navier-Stokes (RANS) equations. Therefore, the same set of equations can be discretized and solved on the same mesh while treating different zones of the geometry with two 
different approaches (detailed RANS for clear fluid zone or coarse mesh porous medium for complex structure zone).

\subsection{Neutronics Sub-solver}

The neutronics sub-solver solves multi-group diffusion Eq. (4) and delayed neutron precursor concentration Eq. (5), including a precursor transport term based on a velocity field $\mathbf{u}$ for the analysis of DNP drift in a liquid fueled reactor (e.g., Molten Salt Reactor):

$$
\begin{gathered}
\frac{1}{v_{i}} \frac{\partial \varphi_{i}}{\partial t}=\nabla \cdot D_{i} \nabla \varphi_{i}+\frac{\left(1-\beta_{t}\right) \chi_{p, i}}{k_{e f f}} \sum_{i=1}^{I} v \Sigma_{f, i} \varphi_{i}-\Sigma_{r, i} \varphi_{i}+\chi_{d, i} \sum_{k=1}^{K} \lambda_{k} C_{k}+S_{s, i} \\
\frac{\partial C_{k}}{\partial t}+\nabla \cdot\left(\boldsymbol{u}_{\boldsymbol{D}} C_{k}\right)=\frac{\beta_{k} \sum_{j} v \Sigma_{f, j} \varphi_{j}}{k_{e f f}}-\lambda_{k} C_{k}
\end{gathered}
$$

In order to calculate the effective delayed neutron fraction in MSRE, the solution of both forward and adjoint multi-group diffusion eigenvalue problems are needed. Since the current version of GeN-Foam only solves the forward neutron flux, a function of computing adjoint neutron flux, according to paper [8], has been implemented. The equations related to the adjoint eigenvalue problems are given in Eqs. (6) and (7):

$$
\begin{gathered}
\nabla \cdot D_{i} \nabla \varphi_{i}^{*}-\Sigma_{r, i} \varphi_{i}^{*}+\sum_{j \neq i} \Sigma_{s, i \rightarrow j} \varphi_{j}^{*}+\frac{1-\beta_{t}}{k_{e f f}} v \Sigma_{f, i} \sum_{i^{\prime}=1}^{I} \chi_{p, i^{\prime}} \varphi_{i^{\prime}}^{*}+\frac{1}{k_{e f f}} v \Sigma_{f, i} \sum_{k=1}^{K} \beta_{k} C_{k}^{*}=0 \\
-\nabla \cdot\left(-\boldsymbol{u}_{\boldsymbol{D}} C_{k}^{*}\right)+\nabla \cdot \frac{v_{T}}{S c_{T}} \nabla C_{k}^{*}-\lambda_{k} C_{k}^{*}+\lambda_{k} \sum_{i=1}^{I} \chi_{d, i} \varphi_{i}^{*}=0
\end{gathered}
$$

After obtaining the forward and adjoint neutron flux distribution, the effective delayed neutron fraction for $\mathrm{k}^{\text {th }} \mathrm{DNP}$ group can be calculated as follows [8-9]:

$$
\beta_{\text {eff }, k}=\frac{\int_{\text {all space }} \sum_{i=1}^{I} \varphi_{i}^{*} \chi_{d, i} \lambda_{k} C_{k}}{\int_{\text {all space }} \sum_{i=1}^{I} \varphi_{i}^{*} \chi_{d, i} \sum_{k^{\prime}=1}^{K} \lambda_{k^{\prime}} C_{k^{\prime}}+\int_{\text {all space }} \sum_{i=1}^{I} \varphi_{i}^{*} \chi_{p, i} \sum_{i^{\prime}=1}^{I} \varphi_{i^{\prime}} v \Sigma_{f, i^{\prime}}},
$$

Note that the value of cross sections, diffusion coefficients and neutron emission spectra are generated by Monte Carlo code Serpent-2, and the number and structure of both neutron energy and DNP groups are based on user's selection. For cross section parametrization in GeN-Foam, eight different perturbated states (i.e., nominal condition, increased/decreased fuel temperature, increased/decreased coolant density, increased/decreased coolant temperature, increased/decreased boron concentration, axially expanded fuel, radial expanded core, and expanded cladding) can be fed to the solver. Linear interpolation between nominal state and perturbed state is applied except the fuel temperature, for which the process can be based either on the logarithm or the square root of temperature, depending on fast or thermal neutron spectrum, respectively.

\section{MOLTEN SALT REACTOR EXPERIMENT MODEL IN GEN-FOAM}

The Molten Salt Reactor Experiment (MSRE) was built at the Oak Ridge National Laboratory and was operated from 1965 to 1969. Its purpose was to demonstrate key features of the molten salt fluid fuel concept and to prove the practicality of the MSR technology. This was the first large-scale, long-term, hightemperature testing performed for a fluid fuel salt, graphite moderator and new nickel-based alloys in a reactor environment. The circulating fuel was a mixture of lithium, beryllium, and zirconium fluoride salts that contained uranium fluorides. Reactor heat was transferred from the fuel salt to a similar coolant salt and was then dissipated to the atmosphere. The MSRE was designed to provide a thermal output of $10 \mathrm{MW}$ [10]. 


\subsection{D-RZ MSRE Geometry and Mesh in GeN-Foam}

A 2D-RZ triangular mesh of MSRE, including the external loop, is generated by Gmsh [11] and shown in Fig. 1. The mesh is used for both thermal-hydraulics and neutronics solvers to simplify the calculations and eliminate possible errors when mapping fields between different meshes. The maximum mesh size is $\sim 3$ $\mathrm{cm}$, with a refinement near the corners and peripheries by reducing it to $\sim 3 \mathrm{~mm}$. The model is divided into 11 regions, and the geometrical sizes of lower plenum, core, upper plenum and downcomer are consistent with MSRE design. However, it is difficult to model the external loop components (e.g., fuel pump, heat exchanger, etc.) in a 2D-RZ coordinate. Therefore, the external loop's geometry is artificial and determined based on the fuel salt volume from report ORNL-TM-728 [10]. The total fuel salt volume in the main stream is $67.3 \mathrm{ft}^{3}\left(1.906 \mathrm{~m}^{3}\right)$, and the volume in each region is listed in Table I. In GeN-Foam, the void (coolant) fraction is adjusted in order to match the fuel salt volume in MSRE design. By conserving fuel volume, fuel residence time can also be matched with MSRE design as long as the volumetric flow rate is same.

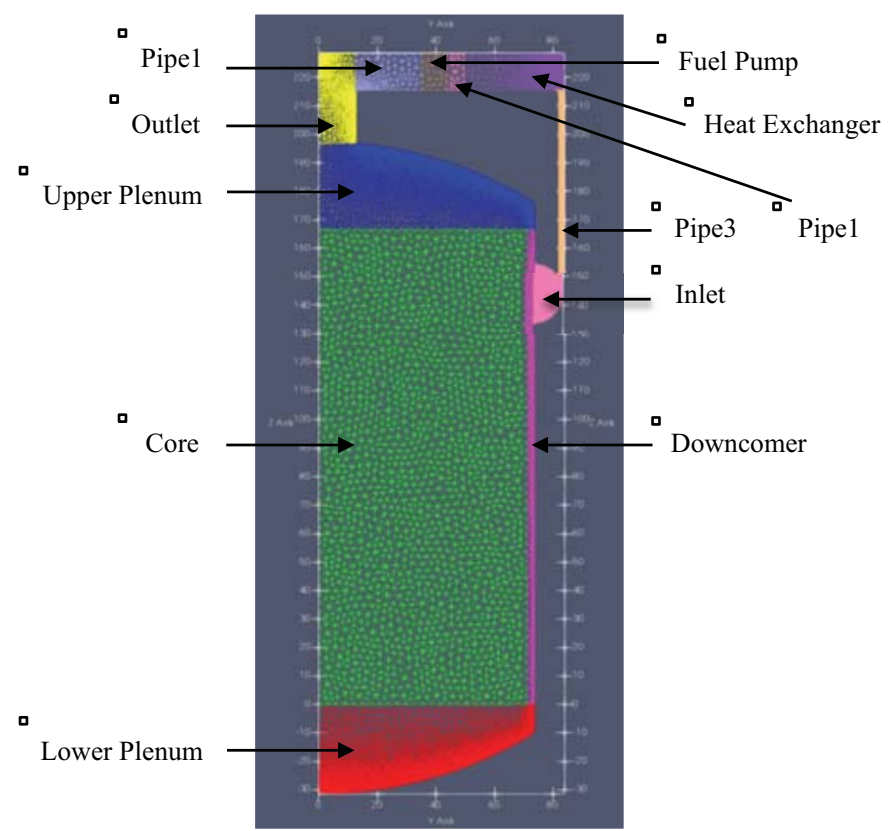

Figure 1. GeN-Foam 2D-RZ MSRE Thermal-hydraulics mesh [unit in $\mathrm{cm}$ ].

Table I. MSRE Fuel Salt Volume and Residence Time [10].

\begin{tabular}{|c|c|c|c|c|}
\hline Regions & $\begin{array}{c}\text { Void Fraction } \\
\text { in GeN-Foam }\end{array}$ & $\begin{array}{c}\text { Fuel Salt Volume in } \\
\text { GeN-Foam Model, } \mathrm{m}^{3}\end{array}$ & $\begin{array}{c}\text { Fuel Salt Volume in } \\
\text { MSRE Design, } \mathrm{m}^{3}\end{array}$ & $\begin{array}{c}\text { Fuel Residence } \\
\text { Time, } \mathrm{s}\end{array}$ \\
\hline Lower Plenum & $79.08 \%$ & $0.358 \times 0.7908=0.283$ & 0.283 & 3.8 \\
\hline Core & $26.77 \%$ & $2.645 \times 0.2677=0.708$ & 0.708 & 9.4 \\
\hline Upper Plenum & $85 \%$ & $0.350 \times 0.85=0.297$ & 0.297 & 3.9 \\
\hline Outlet & $100 \%$ & 0.016 & 0.059 & 0.8 \\
\hline Pipe1 & $100 \%$ & 0.043 & 0.025 & 0.3 \\
\hline Fuel Pump & $100 \%$ & 0.025 & 0.023 & 0.3 \\
\hline Pipe2 & $100 \%$ & 0.023 & 0.173 & 2.3 \\
\hline Heat Exchanger & $94.77 \%$ & $0.182 \times 0.9477=0.173$ & 0.062 & 0.8 \\
\hline Pipe3 & $100 \%$ & 0.062 & 0.275 & 3.6 \\
\hline Inlet & $100 \%$ & 0.082 & 0.192 & \\
\hline Downcomer & $100 \%$ & \multicolumn{2}{|c|}{} \\
\hline
\end{tabular}




\subsection{Thermal-hydraulics Modeling}

The MSRE core region, consisting of 1150 fuel channels and 618 graphite matrixes, is treated as a porous medium. A standard model is applied for coefficients of $\mathrm{k}-\varepsilon$ correlation [1], and Blasius correlation is used for Darcy friction coefficients [12]. The fuel pump is simulated by a momentum force in the direction of flow and able to establish a constant volumetric flow rate of $1200 \mathrm{gpm}\left(0.07571 \mathrm{~m}^{3} / \mathrm{s}\right)$, which equals to the operational flow rate in MSRE. In the heat exchanger zone, Darcy friction factors are adjusted to cause a pressure drop of $\sim 140 \mathrm{kPa}$ at nominal condition. The heat transfer with the secondary loop is simulated by a heat sink proportional to the temperature difference between primary loop and an external fluid at a fixed temperature of $845 \mathrm{~K}$.

In the present work, the fuel salt is assumed to be incompressible, and its thermal-physical properties at 922 $\mathrm{K}$ are given in Table II. Some other assumptions include no control rods modeling, no heat transfer between core and downcomer, no bypass flow from downcomer to upper plenum, and a slip boundary condition for velocity variable on the wall. Besides, during our simulations, a few vortexes are observed when flow enters core from lower plenum. In order to eliminate them, a large flow resistance in the XY plane at the bottom of core is applied. This should not affect our steady-state results as long as the flow rate keeps at $1200 \mathrm{gpm}$. In reality, these vortexes are avoided by the existing of anti-swirl vanes in the lower plenum.

Table II. Fuel Salt Thermal-physical Properties at 922 K [13].

\begin{tabular}{|c|c|}
\hline Parameters & Values \\
\hline Density, $\mathrm{kg} \mathrm{m}^{-3}$ & 2258.6 \\
\hline Specific Heat, $\mathrm{J} \mathrm{kg}^{-1} \mathrm{~K}^{-1}$ & 1967.796 \\
\hline Dynamic Viscosity, Pa s & 0.00785 \\
\hline Prandtl Number & 10.7 \\
\hline
\end{tabular}

\subsection{Neutronics Modeling}

According to the zero-power physics experiments on the MSRE [3], fuel salt composition at the time of criticality is $62.5 \mathrm{LiF}-31.6 \mathrm{BeF}_{2}-5.1 \mathrm{ZrF}_{4}-0.8 \mathrm{UF}_{4}$ (expressed as molar percentages), and the mass fraction of ${ }^{235} \mathrm{U}$ in the salt is $(1.408 \pm 0.007) \mathrm{wt} . \%$. Five different sets of homogenized cross sections (lower head, core, upper head, outlet pipe, and downcomer) are generated by Serpent-2 at $911 \mathrm{~K}$ and $1200 \mathrm{~K}$ as a reference state and a perturbated state, respectively, adopting ENDF/B-VII.1 nuclear data library with pre-defined energy group structures (default 2-group and CASMO 23-group) and six delayed neutron precursor groups (Table III). Their corresponding zones in GeN-Foam model are summarized in Table IV. In addition, two different types of boundary conditions are applied in GeN-Foam neutronics sub-solver. Zero-neutron-flux boundary condition is used for solving forward and adjoint neutron fluxes, and a reflective boundary condition is used for solving concentration and importance of DNPs.

Table III. DNP Groups Decay Constants from Serpent-2 Calculation Using ENDF/B-VII.1.

\begin{tabular}{|c|c|c|c|c|c|c|}
\hline DNP groups & 1 & 2 & 3 & 4 & 5 & 6 \\
\hline Decay Constant, $\mathrm{s}^{-1}$ & $1.3336 \mathrm{E}-02$ & $3.2737 \mathrm{E}-02$ & $1.2079 \mathrm{E}-01$ & $3.0283 \mathrm{E}-01$ & $8.4972 \mathrm{E}-01$ & $2.8537 \mathrm{E}+00$ \\
\hline
\end{tabular}

Table IV. Serpent-2 Cross Sections with their Corresponding Regions in GeN-Foam.

\begin{tabular}{|c|c|}
\hline Serpent Cross Sections & Corresponding Regions in GeN-Foam Model \\
\hline Lower Head & Lower Plenum \\
\hline Core & Core \\
\hline Upper Head & Upper Plenum \\
\hline Outlet Pipe & Outlet, Pipe1, Fuel Pump, Pipe2, Heat Exchanger, and Pipe3 \\
\hline Downcomer & Inlet, Downcomer \\
\hline
\end{tabular}




\section{RESULTS AND DISCUSSIONS}

Two steady-state conditions (static and a constant flow rate of $1200 \mathrm{gpm}$ ) and three transient behaviors (unprotected transient over power, unprotected loss of heat sink, and unprotected loss of flow) are simulated and discussed in the following sections.

\subsection{Steady-State Conditions}

At the nominal flow rate of 1200 gpm, the velocity distritbution is shown in Fig. 2. Also, the values of temperature and pressure at reactor inlet, reactor outlet, heat exchanger inlet and heat exchanger outlet are measured, and they have a good agreement with MSRE design.

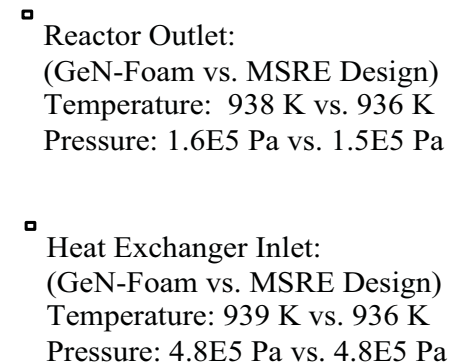

Pressure: 4.8E5 Pa vs. 4.8E5 Pa
Heat Exchanger Outlet:

(GeN-Foam vs. MSRE Design)

Temperature: $910 \mathrm{~K}$ vs. $908 \mathrm{~K}$

Pressure: 3.4E5 Pa vs. 3.4E5 Pa

Reactor Inlet:

(GeN-Foam vs. MSRE Design)

Temperature: $910 \mathrm{~K}$ vs. $908 \mathrm{~K}$

Pressure: 3.5E5 Pa vs. 3.4E5 Pa

Figure 2. Velocity Profile at 1200 gpm.

\subsubsection{Effective multiplication factor ( $\left.k_{\text {eff }}\right)$ and effective delayed neutron fraction ( $\left.\beta_{\text {eff }}\right)$}

The $k_{\text {eff }}$ and $\beta_{\text {eff }}$ calculated by GeN-Foam are summarized in Table V and Table VI, respectively. The results from 2- and 23-energy group structures are very close, less than $25 \mathrm{pcm}$ difference. However, some other numbers of group structures have also been used in calculations, and $\mathrm{k}_{\text {eff }}$ value does not converge until using 23-energy group structures. Therefore, a cancellation of errors occurs with 2-energy group structures.

Table V. keff Values from GeN-Foam.

\begin{tabular}{|c|c|c|c|}
\hline Number of Energy Group & 0 gpm & 1200 gpm & Reactivity change $(\Delta \rho)$ \\
\hline Serpent Default 2 & 1.01232 & 1.00973 & -0.00253 \\
\hline Serpent (CASMO 23) & 1.01243 & 1.00996 & -0.00241 \\
\hline
\end{tabular}

Table VI. $\beta_{\text {eff }}$ Values from GeN-Foam.

\begin{tabular}{|c|c|c|c|c|c|c|c|}
\hline DNP groups & G1 & G2 & G3 & G4 & G5 & G6 & Total \\
\hline Static condition (2-EG) & $2.45 \mathrm{E}-04$ & $1.22 \mathrm{E}-03$ & $1.18 \mathrm{E}-03$ & $2.62 \mathrm{E}-03$ & $1.07 \mathrm{E}-03$ & $4.61 \mathrm{E}-04$ & 0.00680 \\
\hline Static condition (23-EG) & $2.41 \mathrm{E}-04$ & $1.20 \mathrm{E}-03$ & $1.14 \mathrm{E}-03$ & $2.59 \mathrm{E}-03$ & $1.07 \mathrm{E}-03$ & $4.68 \mathrm{E}-04$ & 0.00671 \\
\hline 1200 gpm (2-EG) & $1.16 \mathrm{E}-04$ & $5.09 \mathrm{E}-04$ & $5.84 \mathrm{E}-04$ & $1.80 \mathrm{E}-03$ & $9.62 \mathrm{E}-04$ & $4.54 \mathrm{E}-04$ & 0.00443 \\
\hline 1200 gpm (23-EG) & $1.17 \mathrm{E}-04$ & $5.13 \mathrm{E}-04$ & $5.73 \mathrm{E}-04$ & $1.79 \mathrm{E}-03$ & $9.62 \mathrm{E}-04$ & $4.61 \mathrm{E}-04$ & 0.00441 \\
\hline
\end{tabular}


As the fuel circulates, the $\mathrm{k}_{\text {eff }}$ value drops as expected, and the reactivity change of $-241 \mathrm{pcm}$ agrees with the data of $-212 \mathrm{pcm}$ from report ORNL-4233 [3]. The absolute value of total $\beta_{\text {eff }}$ change of $-230 \mathrm{pcm}$ is smaller than that of $-304 \mathrm{pcm}$ from report ORNL-TM-380 [4]. The discrepancy is due to the simplification in ORNL's MSRE modelling, which the fission of delayed neutron is only considered in the graphitemoderated core region and the contribution of delayed neutrons emitted in the upper and lower plenum is not included. This leads to an underestimation of $\beta_{\text {eff }}$ when fuel is flowing, and thus the change of $\beta_{\text {eff }}$ is overestimated.

\subsubsection{Delayed neutron precursor distribution (DNP group 1, 3, and 6 using 23-energy group)}

The DNP concentrations at static and circulating conditions in MSRE are plotted from Figs. 3 to 5. Note that due to page limits, only DNP group 1, 3, and 6 are presented for demonstration.

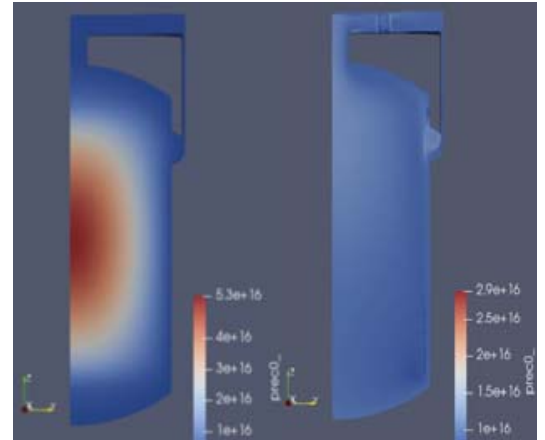

Figure 3. DNP 1 (static vs. flow).

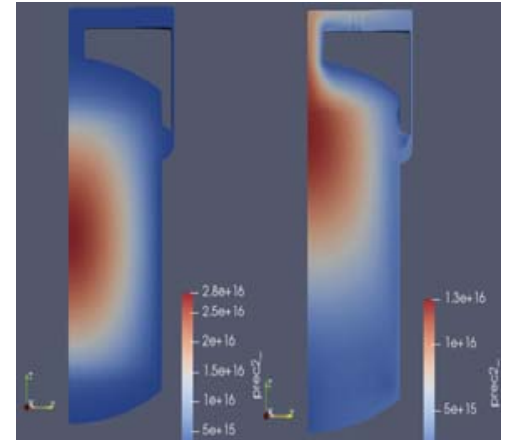

Figure 4. $\mathrm{DNP}_{3}$ (static vs. flow).

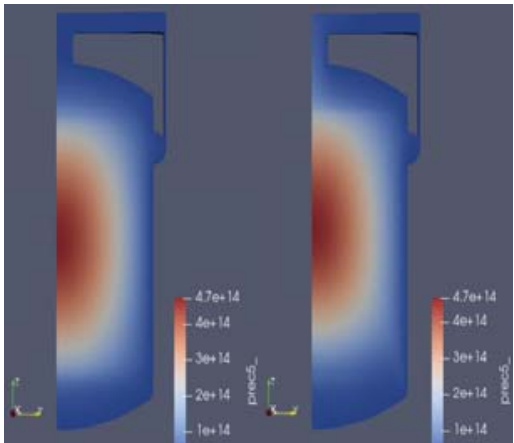

Figure 5. $\mathrm{DNP}_{6}$ (static vs. flow).

For static fuel, the DNP concentrations have a similar shape as the total neutron flux since DNPs are not moving, and their concentrations are proportional to the neutron flux. When the fuel starts to circulate, significant changes can be observed from $\mathrm{DNP}_{1}$ plots. This is because DNP drift as fuel moves, and $\mathrm{DNP}_{1}$ has the smallest decay constant and longest half-life (greater than $50 \mathrm{~s}$ ), so they have time to distribute all over MSRE core and external loop before decaying. As the decay constant increases and half-life decreases, $\mathrm{DNP}_{3}$ has a higher concentration at the top of core and upper plenum region. Finally, for $\mathrm{DNP}_{6}$ with the largest decay constant and shortest half-life (less than $1 \mathrm{~s}$ ), they quickly decay before moving out of the core, resulting in minor changes on the DNP concentration plots.

\subsection{Accident Transients}

In most reactors, there are three main transient initiators, i.e., reactivity insertion, heat exchanger, and primary pump. The following subsections discuss the three possible MSRE responses to the corresponding transient initiators. Note that the main purpose of these analyses is to demonstrate the transient modeling capability of GeN-Foam because a few bugs have been identified and fixed during our study. In addition, even though the steady-state results agree with MSRE design and experimental data, the aforementioned assumptions in section 3.2 and the 2D-RZ geometry may affect the thermal-hydraulics calculations under various transient conditions. Thus, further investigations in this area are required.

\subsubsection{Unprotected transient over power (UTOP)}

An UTOP may occur following a reactivity insertion, which is particularly demanding from a numerical point of view, thus representing a good test for the models. It also gives rise to the maximum power excursion for a given reactivity insertion. For simplicity, three stepwise reactivity insertions have been investigated: $100 \mathrm{pcm}$ (prompt subcritical), $440 \mathrm{pcm}$ (prompt critical), and $500 \mathrm{pcm}$ (prompt supercritical) reactivity insertions. In GeN-Foam, such reactivity insertions are introduced by directly changing $k_{\text {eff }}$ values 
at the beginning of transient calculations, and the resulting power and average fuel temperature profiles are given in Figs. 6 and 7, respectively.

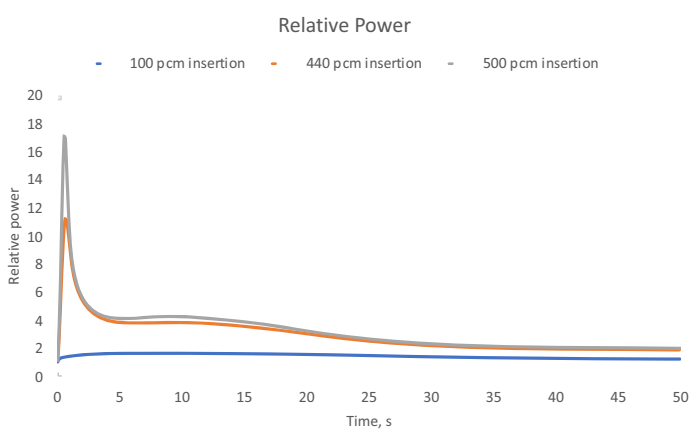

Figure 6. Relative Power in UTOP.

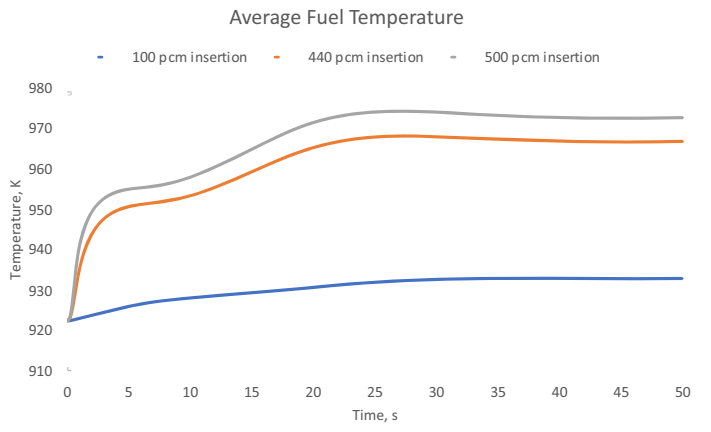

Figure 7. Average Fuel Temperature in UTOP.

It can be observed that by inserting a larger reactivity, a larger power peak occurs at an earlier time. The prompt power increase is triggered by a stepwise reactivity insertion and leads to a rapid temperature increase. Even the reactor is at the prompt critical $\left(\rho=\beta_{\text {eff }}=0.44 \%\right)$ and prompt supercritical $(\rho=0.5 \%>$ $\beta_{\text {eff }}=0.44 \%$ ) states, the consequent negative reactivity insertion due to Doppler effect is able to quickly reduces the power, and the final temperature increase is determined by the amount of initial reactivity inserted.

\subsubsection{Unprotected loss of heat sink (ULOHS)}

An ULOHS may occur following a loss of coolant salt in the reactor secondary loop. For simplicity, the loss of cooling capabilities of heat exchanger is simulated by removing all related heat transfer coefficients in the heat exchanger zone, and the resulting responses are shown in Figs. 8 and 9, respectively.

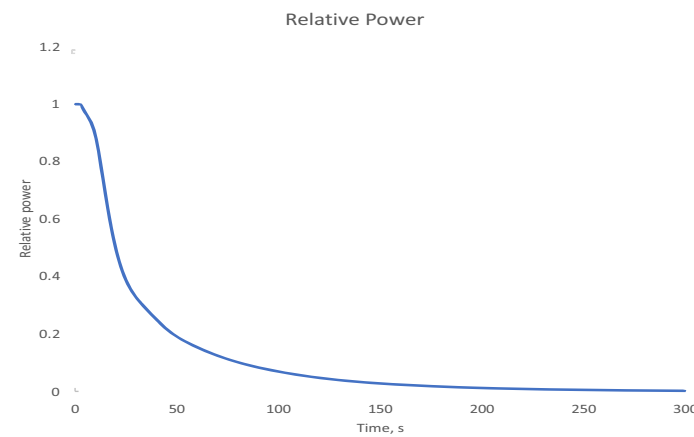

Figure 8. Relative Power in ULOHS.

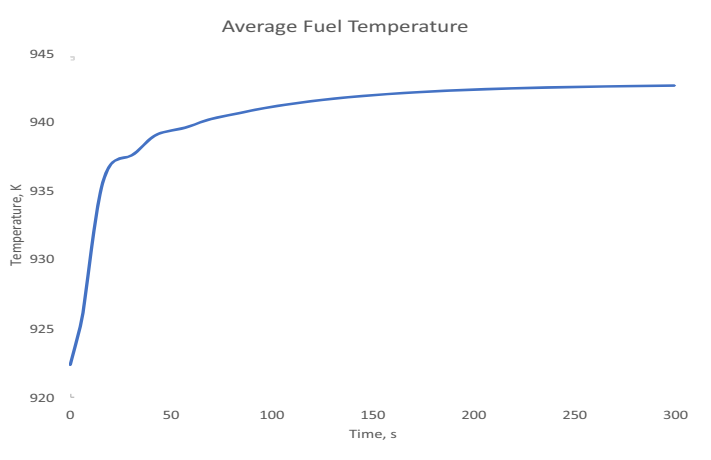

Figure 9. Average Fuel Temperature in ULOHS.

As soon as the cooling capabilities are lost, the fuel salt temperature increases, and thus temperature feedbacks quickly reduce the power and shut down the reactor. The temperature in the primary loop are gradually homogenized because the heat sink is completely removed. In fact, a more realistic scenario would include the effect of decay heat, which will further increase the fuel temperature and lead to a faster and larger power drop.

\subsubsection{Unprotected loss of flow (ULOF)}

An ULOF with a fuel pump coast-down may occur in a reactor following an electricity shortage. In this simulation, the pump force in the primary circuit is assumed to drop exponentially, starting from 1 second with a time constant of $\sim 8$ seconds. After $\sim 27$ seconds, the flow rate drops to $1 / 10$ of the nominal operation 
flow rate in Fig. 10, and the resulting power and average fuel temperature are plotted in Figs. 11 and 12, respectively.

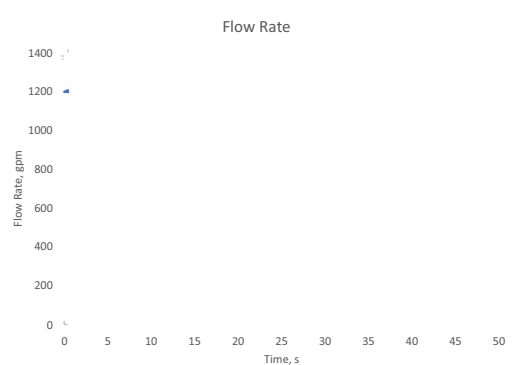

Figure 10. Flow Rate.

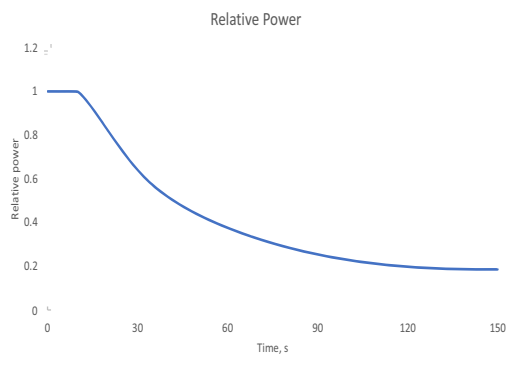

Figure 11. Relative Power.

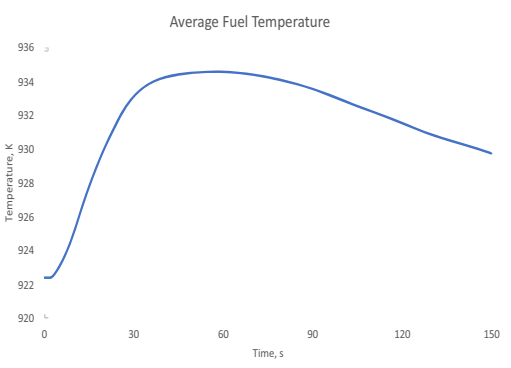

Figure 12. Avg. Fuel Temperature.

As the flow rate decreases, the heat removing capability is reduced and the average fuel temperature initially increases. This temperature increase introduces a negative reactivity due to the Doppler effect and thus reduces power. In fact, at the very beginning of transient ( 1 second to $\sim 10$ seconds), the power does not drop immediately because the speed of DNPs flowing out of the core becomes slower, resulting in a positive reactivity insertion and a minor power rise (not obvious in Fig. 11 due to the plot scale). Finally, the fuel temperature starts to decrease after reaching its peak point when the heat generating rate balances with the heat removing rate.

\section{CONCLUSIONS}

In order to benchmark the delayed neutron precursor drift in MSRE, a 2D-RZ multi-physics model is built in GeN-Foam by conserving the fuel volume and fuel residence time as MSRE design. The cross sections used in GeN-Foam are generated by the Monte Carlo code Serpent-2, adopting ENDF/B-VII.1 nuclear data at reference and perturbated temperatures of $911 \mathrm{~K}$ and $1200 \mathrm{~K}$, respectively. Serpent default 2- and CASMO 23-energy group structures are selected and compared in multi-group neutron diffusion calculations. Although the results are very close, two-energy group structures is not sufficient. For delayed neutron precursor concentration calculations, six DNP groups are chosen. For steady-state calculations (static and fuel flowing at $1200 \mathrm{gpm}$ ) with 23-energy group structures, the $\mathrm{k}_{\text {eff }}$ values are determined to be $\sim 1.01243$ and $\sim 1.00996$, respectively. The reactivity change of $-241 \mathrm{pcm}$ due to fuel circulation is close to MSRE report data of $-212 \mathrm{pcm}$. An adjoint neutron flux solver is also developed and implemented in GeN-Foam to evaluate the effective delayed neutron fractions. The $\beta_{\text {eff, total }}$ is calculated as $0.671 \%$ without DNP drift and $0.441 \%$ with DNP drift, respectively. The absolute value of $\Delta \beta_{\text {eff, total }}$ of $-230 \mathrm{pcm}$ is smaller than the estimated change of $-304 \mathrm{pcm}$ from MSRE report. This discrepancy is due to the omitting of contribution to fission of delayed neutrons emitted in the upper and lower plenum. Moreover, velocity, temperature, pressure, and delayed neutron precursor concentration have been analyzed and shown a reasonable agreement with data from the MSRE. Furthermore, three possible accidental transients (UTOP, ULOHS, and ULOF) have been simulated and discussed. Future improvements may consider modeling of control rods and bypass flow from downcomer to upper plenum region, mesh refinement near walls, and upgrading the model from 2-D to 3-D.

\section{NOMENCLATURE}

$\mathrm{C}_{\mathrm{k}} \quad$ concentration for $\mathrm{k}^{\text {th }}$ DNP group, $\left[\mathrm{m}^{-3}\right]$

$\mathrm{C}_{\mathrm{k}}^{*} \quad$ importance of the $\mathrm{k}^{\text {th }}$ delayed neutron precursor group, $\left[\mathrm{m}^{-2} \mathrm{~s}^{-1}\right]$

$\mathrm{D}_{\mathrm{i}} \quad$ neutron diffusion coefficient for $\mathrm{i}^{\text {th }}$ energy group, [m]

e coolant total energy, $\left[\mathrm{J} \mathrm{kg}^{-1}\right]$

$\mathbf{F}_{\mathrm{g}} \quad$ volumetric force due to gravity, $\left[\mathrm{N} \mathrm{m}^{-3}\right]$ 
$\mathbf{F}_{\text {ss }} \quad$ volumetric force due to the interaction with the sub-scale structure, [ $\left.\mathrm{N} \mathrm{m}^{-3}\right]$

$\mathrm{k}_{\text {eff }}$ effective multiplication factor, [-]

$\mathrm{k}_{\mathrm{T}} \quad$ turbulent conductivity, [ $\mathrm{W} \mathrm{m}^{-1} \mathrm{~K}^{-1}$ ]

$\mathrm{p}$ pressure, $[\mathrm{Pa}]$

$\dot{\mathrm{Q}}_{\mathrm{ss}} \quad$ heat transferred from the sub-scale structure to the fluid, [ $\mathrm{W} \mathrm{m}^{-3}$ ]

$\mathrm{Sc}_{\mathrm{T}} \quad$ turbulent Schmidt number, [-]

$\mathrm{S}_{\mathrm{s}, \mathrm{i}} \quad$ scattering neutron source from neutron energy groups others than $\mathrm{i}^{\text {th }}=\sum_{j \neq i} \sum_{j \rightarrow i} \varphi_{j},\left[\mathrm{~m}^{-3} \mathrm{~s}^{-1}\right]$

u coolant velocity, $\left[\mathrm{m} \mathrm{s}^{-1}\right]$

$\mathbf{u}_{\mathbf{D}} \quad$ Darcy coolant velocity $=\gamma \mathbf{u},\left[\mathrm{m} \mathrm{s}^{-1}\right]$

$\mathrm{v}_{\mathrm{i}} \quad$ average neutron velocity for $\mathrm{i}^{\text {th }}$ energy group, $\left[\mathrm{m} \mathrm{s}^{-1}\right]$

$\beta_{\text {eff, } \mathrm{k}}$ effective delayed neutron fraction for $\mathrm{k}^{\text {th }}$ DNP group, [-]

$\beta_{\text {eff, total }}$ total effective delayed neutron fraction, [-]

$\gamma \quad$ porosity, [-]

$\mu_{\mathrm{T}} \quad$ turbulent dynamic viscosity, [Pa s]

$\lambda_{\mathrm{k}} \quad$ decay constant for $\mathrm{k}^{\text {th }}$ DNP group, $\left[\mathrm{s}^{-1}\right]$

$v \quad$ average number of neutrons per fission, [-]

$v_{\mathrm{T}} \quad$ turbulent kinematic viscosity, $\left[\mathrm{m}^{2} \mathrm{~s}^{-1}\right]$

$\rho \quad$ coolant density, $\left[\mathrm{kg} \mathrm{m}^{-3}\right]$

$\Sigma_{\mathrm{f}, \mathrm{i}} \quad$ fission cross section of $\mathrm{i}^{\text {th }}$ energy group, $\left[\mathrm{m}^{-1}\right]$

$\Sigma_{\mathrm{j} \rightarrow \mathrm{i}} \quad$ group-transfer cross section from $\mathrm{j}^{\text {th }}$ to $\mathrm{i}^{\text {th }}$ energy group, $\left[\mathrm{m}^{-1}\right]$

$\Sigma_{\mathrm{r}, \mathrm{i}} \quad$ removal cross section for $\mathrm{i}^{\text {th }}$ energy group, $\left[\mathrm{m}^{-1}\right]$

$\varphi_{\mathrm{i}} \quad$ neutron flux for $\mathrm{i}^{\text {th }}$ energy group, $\left[\mathrm{m}^{-2} \mathrm{~s}^{-1}\right]$

$\varphi_{\mathrm{i}}^{*} \quad$ adjoint neutron flux for the $\mathrm{i}^{\text {th }}$ energy group, $\left[\mathrm{m}^{-2} \mathrm{~s}^{-1}\right]$

$\chi_{\mathrm{d}, \mathrm{i}} \quad$ delayed neutron yield for $\mathrm{i}^{\text {th }}$ energy group, [-]

$\chi_{\mathrm{p}, \mathrm{i}} \quad$ prompt neutron yield for $\mathrm{i}^{\text {th }}$ energy group, [-]

\section{REFERENCES}

1. C. Fiorina, I. Clifford, M. Aufiero, and K. Mikityuk, "GeN-Foam: A Novel OpenFOAM based Multiphysics Solver for 2D/3D Transient Analysis of Nuclear Reactors," Nuclear Engineering and Design 294, pp. 24-37 (2015).

2. J. Leppänen, et al., "The Serpent Monte Carlo Code: Status, Development and Applications in 2013," Ann. Nucl. Energy 82, pp. 142-150 (2015).

3. B.E. Prince, S.J. Ball, J.R. Engel, P.N. Haubenreich, and T.W. Kerlin, Zero-power Physics Experiments on the Molten-salt Reactor Experiment, Oak Ridge National Laboratory, Oak Ridge, Tennessee, USA (1968).

4. P.N. Haubenreich, Prediction of Effective Yields of Delayed Neutrons in MSRE, Oak Ridge National Laboratory, Oak Ridge, Tennessee, USA (1962).

5. I. Clifford, A Hybrid Coarse and Fine Mesh Solution Method for Prismatic High Temperature Gascooled Reactor Thermal-fluid Analysis, Penn State University, State College, Pennsylvania, USA (2013).

6. R. Saurel, and R. Abgrall, "A Multiphase Godunov Method for Compressible Multifluid and Multiphase Flows," Journal of Computational Physics 150(2), pp. 425-467 (1999).

7. K. Vafai, Handbook of Porous Media, CRC Press, New York, USA (2005).

8. M. Aufiero, et al., "Calculating the Effective Delayed Neutron Fraction in the Molten Salt Fast Reator: Analytical, Deterministic and Monte Carlo Approaches," Annals of Nuclear Energy 65, pp. 78-90 (2014).

9. F. Mattioda, P. Ravetto, and G. Ritter, "Effective Delayed Neutron Fraction of Fluid-fuel Systems," Annals of Nuclear Energy 27(16), pp. 1523-1532 (2000). 
10. R.C. Robertson, MSRE Design and Operations Report Part I Description of Reactor Design, Oak Ridge National Laboratory, Oak Ridge, Tennessee, USA (1965).

11. C. Geuzaine, and J.F. Remacle, "Gmsh: A Three-Dimensional Finite Element Mesh Generator with Built-in Pre- and Post-processing Facilities," International Journal for Numerical Methods in Engineering 79(11), pp. 1309-1331 (2009).

12. J. Bao, Development of the Model for the Multi-physics Analysis of Molten Salt Reactor Experiment using GeN-Foam Code. Paul Scherrer Institute, Villigen, Switzerland (2016).

13. R.J. Kedl, Fluid Dynamic Studies of the Molten-Salt Reactor Experiment (MSRE) Core, Oak Ridge National Laboratory, Oak Ridge, Tennessee, USA (1970). 